\title{
Weak Compactness in Abstract Duality Pairs
}

\author{
Charles Swartz \\ Department of Mathematics, New Mexico State University, Las Cruces, NM 88003, USA \\ Email: cswartz@nmsu.edu
}

\begin{abstract}
Let $E, F$ be (real) vector spaces and $G$ a topological vector space and assume that there is a bilinear map $b: E \times F \rightarrow G$. We call $E, F, G$ an abstract triple (abstract duality pair with respect to $G$ ) and denote it by $(E, F: G)$. We write $b(x, y)=x \cdot y$ for $x \in E, y \in F$. The weakest topology on $E$ such that all of the linear maps $x \rightarrow x \cdot y$ from $E$ into $G$ are continuous for $y \in F$ is denoted by $w(E, F)$. We study sequential compactness and sequential completeness for this topology when $E$ is a space of vector valued, bounded, finitely additive set functions or the space of Bochner or Pettis integrable functions, $F$ is a space of bounded measurable functions, $G$ is a Banach space and the bilinear map is defined via an integral. We also consider vector valued sequence spaces.
\end{abstract}

Keywords: Weak compact, vector valued set functions, Bochner integral, Pettis integral, sequence spaces.

In several recent papers abstract duality pairs or abstract triples have been introduced and used to establish general results such as Orlicz-Pettis Theorems, Banach-Steinhaus Theorems, uniform boundedness and uniform convergence results $[3,4,16,14]$. The results for abstract triples were then used to derive results for locally convex spaces, operator spaces, function spaces and sequence spaces. In this note we derive weak sequential compactness and weak sequential completeness results for abstract triples involving spaces of vector valued set functions and spaces of bounded measurable functions and spaces of vector valued integrable functions and vector valued sequence spaces.

We indicate the notation and assumptions which will be employed. Let $E, F$ be (real) vector spaces and $G$ a topological vector space and assume that there is a bilinear map $b: E \times F \rightarrow G$. We call $E, F, G$ an abstract triple (abstract duality pair with respect to $G$ ) and denote it by $(E, F: G)$. More general abstract triples are considered in $[3,4,16]$. We write $b(x, y)=x \cdot y$ for $x \in E, y \in F$. The weakest topology on $E$ such that all of the linear maps $x \rightarrow x \cdot y$ from $E$ into $G$ are continuous for $y \in F$ is denoted by $w(E, F)$. We study sequential compactness and sequential completeness for this topology when $E$ is a space of vector valued, bounded, finitely additive set functions or the space of Bochner or Pettis integrable functions, $F$ is a space of bounded measurable functions, $G$ is a Banach space and the bilinear map is defined via an integral.

In what follows $X$ will denote a Banach space and $\Sigma$ a $\sigma$-algebra of subsets of a set $S$. We will require some integration results for bounded, measurable functions with respect to bounded, finitely additive, scalar set functions and bounded, finitely additive $X$-valued set functions.

First, assume $\nu: \Sigma \rightarrow \mathbb{R}$ is bounded and finitely additive. The variation of $\nu$ is denoted by $|\nu|[14]$. If $f: S \rightarrow \mathbb{R}$ is a $\Sigma$ simple function, $f=\sum_{k=1}^{n} a_{k} \chi_{A_{k}}$, the integral of $f$ with respect to $\nu$ is defined to be

$$
\int_{A} f \mathrm{~d} \nu=\sum_{k=1}^{n} a_{k} \nu\left(A_{k}\right)
$$

the integral is independent of the representation of $F$ as a simple function. Note

$$
\text { (*) }\left|\int_{A} f \mathrm{~d} \nu\right| \leq \sum_{k=1}^{n}\left|a_{k}\right|\left|\nu\left(A_{k} \cap A\right)\right| \leq\|f\|_{\infty}|\nu|(A)
$$

for $A \in \Sigma$, where $\|\cdot\|_{\infty}$ denotes the sup-norm. If $f: S \rightarrow \mathbb{R}$ is bounded and $\Sigma$ measurable and $\left\{f_{k}\right\}$ is a sequence of simple functions converging uniformly to $f$, the integral of $f$ with respect to $\nu$ is defined to be

$$
\int_{A} f \mathrm{~d} \nu=\lim _{k} \int_{A} f_{k} \mathrm{~d} \nu
$$


note $\left\{\int_{A} f_{k} \mathrm{~d} \nu\right\}$ is Cauchy by $(*)$ so the limit exists, the integral is independent of the sequence $\left\{f_{k}\right\}$ and the inequality $(*)$ still holds for $f$.

Next, we define the integral of a scalar valued function with respect to a finitely additive, bounded set function. Let $m: \sum \rightarrow X$ be finitely additive and bounded and let $f: S \rightarrow \mathbb{R}$ be $\Sigma$ measurable.

Definition 1. $f$ is $m$ integrable if for each $x^{\prime} \in X^{\prime}, f$ is $x^{\prime} m$ integrable and for each $A \in \Sigma$ there exists $x_{A} \in X$ such that $\int_{A} f \mathrm{~d} x^{\prime} m=x^{\prime}\left(x_{A}\right)$. We write $x_{A}=\int_{A} f \mathrm{~d} m$ so $x^{\prime}\left(\int_{A} f \mathrm{~d} m\right)=\int_{A} f \mathrm{~d} x^{\prime} m$.

We define the semi-variation of $m$ in order to obtain the analogue of $\left(^{*}\right)$ for the integral.

Definition 2. The semi-variation of $m$ is defined by

$$
\|m\|(A)=\sup \left\{\left\|\sum_{k=1}^{n} t_{k} m\left(A_{k}\right)\right\|:\left|t_{k}\right| \leq 1,\left\{A_{k}\right\} \subset \Sigma \text { a partition of } A\right\} .
$$

Note that

$$
\begin{aligned}
\|m\|(A) & =\sup \left\{\left|\sum_{k=1}^{n} t_{k} x^{\prime} m\left(A_{k}\right)\right|:\left\|x^{\prime}\right\| \leq 1,\left|t_{k}\right| \leq 1,\left\{A_{k}\right\} \subset \Sigma \text { a partition of } A\right\} \\
& =\sup \left\{\left|x^{\prime} m\right|(A):\left\|x^{\prime}\right\| \leq 1\right\}
\end{aligned}
$$

and also

$$
(* *)\|m(A)\| \leq\|m\|(A) \leq 4 \sup \{\|m(B)\|: B \subset A, B \in \Sigma\} ;
$$

see [5] IV.10.4.

Proposition 3. Let $f: S \rightarrow \mathbb{R}$ be bounded and $\Sigma$ measurable. Then $f$ is $m$ integrable with

$$
\text { (\#) }\left\|\int_{A} f \mathrm{~d} m\right\| \leq\|f\|_{\infty}\|m\|(A)
$$

Proof. First assume that $f$ is $m$ integrable. Then

$$
\begin{aligned}
\left\|\int_{A} f \mathrm{~d} m\right\| & =\sup \left\{\left|\int_{A} f \mathrm{~d} x^{\prime} m\right|:\left\|x^{\prime}\right\| \leq 1\right\} \leq \sup \left\{\int_{A}|f| d\left|x^{\prime} m\right|:\left\|x^{\prime}\right\| \leq 1\right\} \\
& \leq\|f\|_{\infty} \sup \left\{\left|x^{\prime} m\right|(A):\left\|x^{\prime}\right\| \leq 1\right\}=\|f\|_{\infty}\|m\|(A) .
\end{aligned}
$$

so (\#) holds.

Next pick a sequence of simple functions $\left\{g_{j}\right\}$ which converge uniformly to $f$. Clearly every simple function is $m$ integrable so (\#) holds for simple functions. From (\#),

$$
\left\|\int_{A} g_{j} \mathrm{~d} m-\int_{A} g_{k} \mathrm{~d} m\right\| \leq\left\|g_{j}-g_{k}\right\|_{\infty}\|m\|(A)
$$

so $\left\{\int_{A} g_{k} \mathrm{~d} m\right\}$ is Cauchy. Let $x_{A}=\lim \int_{A} g_{k} \mathrm{~d} m$. We claim $x_{A}=\int_{A} f \mathrm{~d} m$. Let $x^{\prime} \in X^{\prime}$. Then

$$
x^{\prime}\left(x_{A}\right)=\lim x^{\prime} \int_{A} g_{k} \mathrm{~d} m=\int_{A} g_{k} \mathrm{~d} x^{\prime} m=\int_{A} f \mathrm{~d} x^{\prime} m
$$

justifying the claim. Thus, $f$ is $m$ integrable with (\#) holding.

We establish a lemma which will be used in the sequel. Let $B(\Sigma)$ be the space of all bounded, $\Sigma$ measurable functions with the sup-norm $\|\cdot\|_{\infty}$ and let $b a(\Sigma, X)$ be the space of all bounded, finitely additive $m: \Sigma \rightarrow X$ with the semi-variation norm, $\|m\|=\|m\|(S)$.

Lemma 4. Suppose $m_{k} \in b a(\Sigma, X)$ and $m(A)=\lim m_{k}(A)$ exists for every $A \in \Sigma$. Then

(i) $m \in b a(\Sigma, X)$ and

(ii) for every $f \in B(\Sigma), \lim \int_{A} f \mathrm{~d} m_{k}=\int_{A} f \mathrm{~d} m$. 
Proof. (i): That $m$ is finitely additive is clear and $m$ is bounded by the Nikodym Boundedness Theorem $[6]$.

(ii): Let $\epsilon>0$. Pick $g$ simple such that $\|f-g\|_{\infty} \leq \epsilon$ and let

$$
M=\sup \left\{\left\|m_{k}(A)\right\|: k \in \mathbb{N}, A \in \Sigma\right\}
$$

note $M<\infty$ by the Nikodym Boundedness Theorem. Pick $n$ such that $\left\|\int_{A} g \mathrm{~d} m_{k}-\int_{A} g \mathrm{~d} m\right\| \leq \epsilon$ for $k \geq n$ (hypothesis). For $k \geq n$,

$$
\begin{aligned}
& \left\|\int_{A} f \mathrm{~d} m_{k}-\int_{A} f \mathrm{~d} m\right\| \\
\leq & \left\|\int_{A}(f-g) \mathrm{d} m_{k}\right\|+\left\|\int_{A}(f-g) \mathrm{d} m\right\| \\
& +\left\|\int_{A} g \mathrm{~d} m_{k}-\int_{A} g \mathrm{~d} m\right\| \\
\leq & \|f-g\|_{\infty}\left\|m_{k}\right\|(A)+\|f-g\|_{\infty}\|m\|(A)+\epsilon \\
\leq & \epsilon 8 M+\epsilon
\end{aligned}
$$

by $(* *)$.

Let $S(\Sigma)$ be the subspace of $B(\Sigma)$ consisting of the simple functions. Consider the abstract triple $(b a(\Sigma, X), S(\Sigma): X)$ under the integration map $(m, f) \rightarrow \int_{S} f \mathrm{~d} m$. The hypothesis of Lemma 4 asserts that the sequence $\left\{m_{k}\right\}$ is

$$
w(b a(\Sigma, X), S(\Sigma))
$$

Cauchy. Now consider the abstract triple $(b a(\Sigma, X), S(\Sigma): X)$ under the integration map. The conclusion of Lemma 4 is that there exists $m \in b a(\Sigma, X)$ such that the sequence $\left\{m_{k}\right\}$ converges to $m$ in the stronger topology $w(b a(\Sigma, X), B(\Sigma))$. Thus, Lemma 4 can be viewed as a "Schur-type" result; i.e., in $l^{1}$ a sequence which is Cauchy in the weak topology actually is convergent in the norm topology[15].

We consider compactness results for spaces of vector valued set functions. A subset $K$ of a topological vector space $E$ is relatively sequentially compact if every sequence $\left\{x_{k}\right\} \subset K$ has a subsequence $\left\{x_{n_{k}}\right\}$ which converges to an element in $E ; K$ is conditionally sequentially compact if every sequence $\left\{x_{k}\right\} \subset K$ has a subsequence $\left\{x_{n_{k}}\right\}$ which is Cauchy.

We first consider a boundedness result.

Proposition 5. Suppose $K \subset b a(\Sigma, X)$ is $w(b a(\Sigma, X), S(\Sigma))$ conditionally sequentially compact. Then $K$ is norm bounded in ba $(\Sigma, X)$.

Proof. $\{m(A): m \in K, A \in \Sigma\}$ is bounded iff $\left\{m_{k}\left(A_{k}\right)\right\}$ is bounded for every $\left\{m_{k}\right\} \subset K$ and every pairwise disjoint sequence $\left\{A_{k}\right\} \subset \Sigma[13]$. By $w(b a(\Sigma, X), S(\Sigma))$ conditionally sequentially compactness we may assume that $\lim m_{k}(A)$ exists for every $A \in \Sigma$. Then $\left\{m_{k}(A)\right\}$ is bounded for every $A \in \Sigma$ and $\left\{m_{k}\left(A_{k}\right)\right\}$ is bounded by the Nikodym Boundedness Theorem. $K$ is norm bounded by $(* *)$.

Definition 6. A finitely additive set function $m: \Sigma \rightarrow X$ is strongly additive (strongly bounded) if the series $\sum_{k=1}^{\infty} m\left(A_{k}\right)$ converges for every pairwise disjoint sequence $\left\{A_{k}\right\} \subset \Sigma$.

A strongly additive set function is bounded[6]. Let $\operatorname{sba}(\Sigma, X)$ be the space of all strongly bounded elements of $b a(\Sigma, X)$. A subset $M \subset \operatorname{sba}(\Sigma, X)$ is uniformly strongly additive if the series $\sum_{k=1}^{\infty} m\left(A_{k}\right)$ converge uniformly for $m \in M$ and every pairwise disjoint sequence $\left\{A_{k}\right\} \subset \Sigma$.

Consider the abstract triple $(s b a(\Sigma, X), S(\Sigma): X)$ under the integration map $(m, f) \rightarrow \int_{S} f \mathrm{~d} m$.

Proposition 7. If $K \subset \operatorname{sba}(\Sigma, X)$ is $w(\operatorname{sba}(\Sigma, X), S(\Sigma))$ conditionally sequentially compact, then

(I) $K$ is uniformly strongly additive. 
Proof. If the conclusion fails, there exist $m_{k} \in K,\left\{A_{k}\right\} \subset \Sigma$ pairwise disjoint, an increasing sequence of intervals, $\left\{I_{k}\right\}$, in $\mathbb{N}$ and $\epsilon>0$ such that

$$
\text { (\&) }\left\|\sum_{j \in I_{k}} m_{k}\left(A_{j}\right)\right\|>\epsilon .
$$

By $w(\operatorname{sba}(\Sigma, X), S(\Sigma))$ conditionally sequentially compactness we may assume $\lim m_{k}(A)=m(A)$ exists for every $A \in \Sigma$. By the Nikodym Convergence Theorem[6], $\left\{m_{k}\right\}$ is uniformly strongly additive contradicting (\&).

Proposition 8. Suppose $K \subset b a(\Sigma, X)$ is $w(b a(\Sigma, X), B(\Sigma))$ conditionally (relatively) sequentially compact. Then for every $f \in B(\Sigma)$,

$$
\begin{aligned}
(I I) K_{f}= & \left\{\int_{S} f \mathrm{~d} m: m \in K\right\} \text { is }\|\cdot\| \text { conditionally } \\
& \text { (relatively) sequentially compact. }
\end{aligned}
$$

In particular, for every $A \in \Sigma$,

$$
\begin{aligned}
(I I I) K_{A}= & \{m(A): m \in K\} \text { is }\|\cdot\| \text { conditionally } \\
& (\text { relatively) sequentially compact. }
\end{aligned}
$$

Proof. The linear map from $m \rightarrow \int_{S} f \mathrm{~d} m$ from $b a(\Sigma, X) \rightarrow X$ is

$$
w(b a(\Sigma, X), B(\Sigma))-\|\cdot\|
$$

sequentially continuous so the result follows.

Conditions (I),(II) and (III) give necessary conditions for $w(s b a(\Sigma, X), B(\Sigma))$ relative sequential compactness. We now consider sufficient conditions.

Theorem 9. Let $K \subset \operatorname{sba}(\Sigma, X)$. Assume that $\Sigma$ is generated by a countable algebra $\mathcal{A}$. Then conditions (I) and (III) imply that $K$ is $w(s b a(\Sigma, X), B(\Sigma))$ relatively sequentially compact.

Proof. Let $\left\{m_{k}\right\} \subset \operatorname{sba}(\Sigma, X)$. By (III) and the diagonalization method[7], there exists a subsequence $\left\{q_{k}\right\}$ of $\left\{m_{k}\right\}$ such that $\|\cdot\|-\lim q_{k}(A)=m(A)$ exists for every $A \in \mathcal{A}$. We claim that $\|\cdot\|-\lim q_{k}(A)=m(A)$ exists for every $A \in \Sigma$. For this let

$$
\Sigma_{1}=\left\{A \in \sum:\|\cdot\|-\lim _{k} q_{k}(A)=m(A) \text { exists }\right\}
$$

Then $\mathcal{A} \subset \Sigma_{1}$ and we claim that $\Sigma_{1}$ is a monotone class. Suppose $B_{j} \in \Sigma$ and $B_{j} \uparrow B$. By definition of $\Sigma_{1},\|\cdot\|-\lim _{k} q_{k}\left(B_{j}\right)=m\left(B_{j}\right)$ exists for every $j$. Now $B_{j}=B_{1} \cup\left(\cup_{i=1}^{j-1}\left(B_{i+1} \backslash B_{i}\right)\right)$ so by (I), $\|\cdot\|-\lim _{j} q_{k}\left(B_{j}\right)=z_{k}$ exists uniformly for $k \in \mathbb{N}$. By the Iterated Limit Theorem[5],

$$
\lim _{j} \lim _{k} q_{k}\left(B_{j}\right)=\lim _{k} \lim _{j} q_{k}\left(B_{j}\right)=\lim _{k} z_{k}(B)=\lim _{j} m\left(B_{j}\right),
$$

where all limits are with respect to the norm. Hence, $B \in \Sigma_{1}$. A similar computation holds for decreasing sequences from $\Sigma_{1}$. Hence, $\Sigma_{1}$ is a monotone class and the Monotone Class Theorem[14] implies that $\Sigma_{1}=\Sigma$ justifying the claim. By the Nikodym Convergence Theorem $m \in \operatorname{sba}(\Sigma, X)$. Now by Lemma 4

$$
\lim \int_{S} f \mathrm{~d} q_{k}=\int_{S} f \mathrm{~d} m
$$

holds for every $f \in B(\Sigma)$ so $q_{k} \rightarrow m$ in $w(\operatorname{sba}(\Sigma, X), B(\Sigma))$. 
Corollary 10. Let $K \subset \operatorname{sba}(\Sigma, X)$. Assume that $\Sigma$ is generated by a countable algebra $\mathcal{A}$. Then $K$ is $w(\operatorname{sba}(\Sigma, X), B(\Sigma))$ relatively sequentially compact iff (I) and (III) hold.

In the remarks below assume that $\Sigma$ is generated by a countable algebra $\mathcal{A}$.

The same proof shows that if $K \subset b a(\Sigma, X)$ satisfies (I) and (III), then $K$ is $w(b a(\Sigma, X), B(\Sigma))$ relatively sequentially compact. For if

$$
\|\cdot\|-\lim q_{k}(A)=m(A)
$$

exists for every $A \in \Sigma$, then $m \in b a(\Sigma, X)$ by applying the Nikodym Boundedness Theorem in place of the Nikodym Convergence Theorem.

Similarly, if $c a(\Sigma, X)$ denotes the space of all countably additive members of $s b a(\Sigma, X)$ and $K \subset$ $c a(\Sigma, X)$, then $K$ is $w(c a(\Sigma, X), B(\Sigma))$ relatively sequentially compact iff (I) and (III) hold. For the limit of countably additive set function is countably additive by The Nikodym Convergence Theorem[5].

Let $\lambda: \Sigma \rightarrow[0, \infty)$ be a measure and let $c a(\Sigma, X: \lambda)$ be the elements of $c a(\Sigma, X)$ which are absolutely continuous with respect to $\lambda$. Then $K$ is $w(c a(\Sigma, X: \lambda), B(\Sigma))$ is relatively sequentially compact iff (I) and (III) hold. For if

$$
\|\cdot\|-\lim q_{k}(A)=m(A)
$$

exists for every $A \in \Sigma$, then $m \in c a(\Sigma, X: \lambda)$ by the Vitali-Hahn-Saks Theorem[6].

It is worthwhile observing that the methods above imply the weak sequential completeness for the topologies $w(Y, Z)$ when $Y=b a(\Sigma, X), \operatorname{sba}(\Sigma, X), c a(\Sigma, X), c a(\Sigma, X: \lambda)$ and $Z=S(\Sigma), B(\Sigma)$.

We next consider abstract triples of vector valued integrable functions. Let $L^{1}(\lambda, X)$ be the space of Bochner $\lambda$ integrable $X$ valued functions [1,6] with the complete norm $\|f\|_{1}=\int_{S}\|f(s)\| \mathrm{d} \lambda$ and consider the triples $\left(L^{1}(\lambda, X), S(\Sigma): X\right)$ and $\left(L^{1}(\lambda, X), L^{\infty}(\lambda): X\right)$ under the integration map $(f, g) \rightarrow \int_{S} g f \mathrm{~d} \lambda$. We establish the analogues of Propositions 7 and 8 for this triple.

Proposition 11. Let $K \subset L^{1}(\lambda, X)$ and suppose $K$ is $w\left(L^{1}(\lambda, X), S(\Sigma)\right)$ conditionally sequentially compact. Then $\left\{\int f \mathrm{~d} \lambda: f \in K\right\}$ is uniformly countably additive.

Proof. If the conclusion fails, there exist $\left\{f_{k}\right\} \subset K,\left\{A_{k}\right\} \subset \Sigma$ pairwise disjoint, an increasing sequence of intervals $\left\{I_{k}\right\}$ and $\epsilon>0$ such that

$$
\text { (*) }\left\|\int_{\cup_{j \in I_{k}} A_{j}} f_{k} \mathrm{~d} \lambda\right\|>\epsilon .
$$

We may assume that $\lim _{k} \int_{A} f_{k} \mathrm{~d} \lambda$ exists for every $A \in \Sigma$. The Nikodym Convergence Theorem asserts that the measures $\left\{\int f_{k} \mathrm{~d} \lambda: f \in K\right\}$ are uniformly countably additive. This contradicts $\left(^{*}\right)$.

Proposition 12. Let $K \subset L^{1}(\lambda, X)$ and suppose $K$ is $w\left(L^{1}(\lambda, X), S(\Sigma)\right)$ conditionally sequentially compact. Then for every $A \in \Sigma, K_{A}=\left\{\int_{A} f \mathrm{~d} \lambda: f \in K\right\}$ is $\|\cdot\|$ conditionally sequentially compact. If $K$ is $w\left(L^{1}(\lambda, X), L^{\infty}(\lambda)\right)$ conditionally sequentially compact, then $K_{h}=\left\{\int_{S} h f \mathrm{~d} \lambda: f \in K\right\}$ is $\|\cdot\|$ conditionally sequentially compact for every $h \in L^{\infty}(\lambda)$.

Proof. The linear map $H: L^{1}(\lambda, X) \rightarrow X, f \rightarrow \int_{S} h f \mathrm{~d} \lambda$, is $\omega\left(L^{1}(\lambda, X), L^{\infty}(\lambda)-X\right.$ continuous. The result follows.

The conclusions in Propositions 11 and 12 are necessary conditions for weak conditional sequential compactness. As in Theorem 9 we consider sufficient conditions.

Theorem 13. Let $K \subset L^{1}(\lambda, X)$ be bounded. Assume that $\Sigma$ is generated by a countable algebra $\mathcal{A}$. If $\left\{\int f \mathrm{~d} \lambda: f \in K\right\}$ is uniformly countably additive and $K_{h}=\left\{\int_{S} h f \mathrm{~d} \lambda: f \in K\right\}$ is $\|\cdot\|$ conditionally sequentially compact for every $h \in L^{\infty}(\lambda)$, then $K$ is $w\left(L^{1}(\lambda, X), L^{\infty}(\lambda)\right)$ conditionally sequentially compact. If $X$ has the Radon-Nikodym property with respect to $\lambda[6]$, then $K$ is $w\left(L^{1}(\lambda, X), L^{\infty}(\lambda)\right)$ relatively sequentially compact. 
Proof. Let $\left\{f_{k}\right\} \subset K$ and set $M=\sup \left\{\left\|f_{k}\right\|_{1}: k\right\}$. As in the proof of Theorem 9 we may assume that there exists a subsequence $\left\{g_{k}\right\}$ of $\left\{f_{k}\right\}$ such that $\lim \int_{A} g_{k} \mathrm{~d} \lambda$ exists for every $A \in \Sigma$.

Now we claim that $\lim \int_{S} h g_{k} \mathrm{~d} \lambda$ exists for every $h \in L^{\infty}(\lambda)$. The linear maps $G_{k}: L^{\infty}(\lambda) \rightarrow X$, $G_{k}(h)=\int_{S} h g_{k} \mathrm{~d} \lambda$, are continuous and uniformly bounded since $\left\|G_{k}(h)\right\| \leq\left\|g_{k}\right\|_{1}\|h\|_{\infty} \leq M\|h\|_{\infty}$. Now $\lim \int_{S} h g_{k} \mathrm{~d} \lambda$ exists for every simple function $h$ and the simple functions are dense in $L^{\infty}(\lambda)$ so $\lim \int_{S}^{\infty} h g_{k} \mathrm{~d} \lambda$ exists for every $h \in L^{\infty}(\lambda)$ by the uniform boundedness of the $\left\{G_{k}\right\}$. Hence, $K$ is $w\left(L^{1}(\lambda, X), L^{\infty}(\lambda)\right)$ conditionally sequentially compact.

Assume the Radon-Nikodym property. Set $m(A)=\lim \int_{A} g_{k} \mathrm{~d} \lambda$ for $A \in \Sigma$. Then $m$ is countably additive by the Nikodym Convergence Theorem and we claim that $m$ has bounded variation. Let $\left\{A_{j}: j=1, \ldots, n\right\} \subset \Sigma$ be a partition of $S$. Then

$$
\sum_{j=1}^{n}\left\|m\left(A_{j}\right)\right\|=\lim _{k} \sum_{j=1}^{n}\left\|\int_{A} g_{k} \mathrm{~d} \lambda\right\| \leq \lim \sup _{k} \sum_{j=1}^{n} \int_{A_{j}}\left\|g_{k}(s)\right\| \mathrm{d} \lambda(s) \leq M
$$

so the claim is justified. By the Radon-Nikodym property there exists $g \in L^{1}(\lambda, X)$ such that $m(A)=$ $\int_{A} g \mathrm{~d} \lambda$ for $A \in \Sigma$. By what was established above $g_{k} \rightarrow g$ in $w\left(L^{1}(\lambda, X), L^{\infty}(\lambda)\right)$ and $K$ is $w\left(L^{1}(\lambda, X), L^{\infty}(\lambda)\right)$ relatively sequentially compact.

For the scalar version of these results for $L^{1}(\lambda)$, see [5].

Next, we consider abstract triples involving the Pettis integral. A function $f: S \rightarrow X$ is Pettis integrable with respect to $\lambda$ if the function $x^{\prime} f$ is $\lambda$ integrable for every $x^{\prime} \in X^{\prime}$ and for every $A \in \Sigma$ there exists $x_{A} \in X$ such that $\int_{A} x^{\prime} f \mathrm{~d} \lambda=x^{\prime}\left(x_{A}\right) ; x_{A}$ is called the Pettis integral of $f$ and is denoted by $\int_{A} f \mathrm{~d} \lambda$. For the properties of the Pettis integral see $[6,11]$. In particular, the indefinite integral of a Pettis integrable function is countably additive. Let $P(\lambda, X)$ be the space of Pettis integrable functions; this space has two equivalent norms, $\|f\|_{P}=\sup \left\{\left|\int_{S}\right| x^{\prime} f|\mathrm{~d} \lambda|:\left\|x^{\prime}\right\| \leq 1\right\}$ and $\|f\|_{P}^{\prime}=\sup \left\{\left\|\int_{A} f \mathrm{~d} \lambda\right\|: A \in \Sigma\right\}$ which in general are not complete. Consider the abstract triples $(P(\lambda, X), S(\Sigma): X),(P(\lambda, X), B(\Sigma): X)$ under the integral map $(f, g) \rightarrow \int_{S} g f \mathrm{~d} \lambda[6]$ for the existence of the integral ). The analogue of Proposition 11 for this triple is established as in the case of the Bochner integral above.

Proposition 14. Let $K \subset P(\lambda, X)$ and suppose $K$ is $w(P(\lambda, X), S(\Sigma))$ conditionally sequentially compact. Then $\left\{\int f \mathrm{~d} \lambda: f \in K\right\}$ is uniformly countably additive.

Proposition 15. Let $K \subset P(\lambda, X)$ and suppose $K$ is $w(P(\lambda, X), S(\Sigma))$ conditionally sequentially compact. Then for every $A \in \Sigma, K_{A}=\left\{\int_{A} f \mathrm{~d} \lambda: f \in K\right\}$ is $\|\cdot\|$ conditionally sequentially compact. If $K$ is $w(P(\lambda, X), B(\Sigma))$ conditionally sequentially compact, then $K_{h}=\left\{\int_{S} h f \mathrm{~d} \lambda: f \in K\right\}$ is $\|\cdot\|$ conditionally sequentially compact for every $h \in B(\Sigma)$.

Proof. The linear map $H: P(\lambda, X) \rightarrow X, f \rightarrow \int_{S} h f \mathrm{~d} \lambda$, is $\omega(P(\lambda, X), B(\Sigma))-X$ continuous. The result follows.

The conclusions in Propositions 14 and 15 are necessary conditions for weak conditional sequential compactness. As in Theorem 13 we consider sufficient conditions.

Theorem 16. Let $K \subset P(\lambda, X)$. Assume that $\Sigma$ is generated by a countable algebra $\mathcal{A}$. If $\left\{\int f \mathrm{~d} \lambda: f \in K\right\}$ is uniformly countably additive and $K_{h}=\left\{\int_{S} h f \mathrm{~d} \lambda: f \in K\right\}$ is $\|\cdot\|$ conditionally sequentially compact for every $h \in B(\Sigma)$, then $K$ is $w(P(\lambda, X), B(\Sigma))$ conditionally sequentially compact.

Proof. Let $\left\{f_{k}\right\} \subset K$. As in the proof of Theorem 9 we may assume that there exists a subsequence $\left\{g_{k}\right\}$ of $\left\{f_{k}\right\}$ such that $\lim \int_{A} g_{k} \mathrm{~d} \lambda$ exists for every $A \in \Sigma$.

Now we claim that $\lim \int_{S} h g_{k} \mathrm{~d} \lambda$ exists for every $h \in B(\Sigma)$. Since $K_{h}=\left\{\int_{S} h f \mathrm{~d} \lambda: f \in K\right\}$ is $\|\cdot\|$ conditionally sequentially compact, every subsequence of $\left\{g_{k}\right\}$ has a subsequence $\left\{g_{n_{k}}\right\}$ such that $\lim \int_{S} h g_{n_{k}} \mathrm{~d} \lambda$ exists. Since $X$ is complete, $\lim \int_{S} h g_{k} \mathrm{~d} \lambda$ exists. Thus, $K$ is $w(P(\lambda, X), B(\Sigma))$ conditionally sequentially compact.

In the proof of Theorem 16 we have that $\lim \int_{A} g_{k} \mathrm{~d} \lambda=m(A)$ exists for every $A \in \Sigma$, but a lack of Radon-Nikodym Theorems for the Pettis integral means that we do not have a Pettis integrable function 
$g$ such that $\int . g \mathrm{~d} \lambda=m(\cdot)[11]$. This is the reason for the condition sequential compactness statement instead of a relative sequential compactness statement in the theorem.

Results similar to those above can also be established for the space of Dunford integrable functions $[6,11]$ for this integral).

We consider triples involving vector valued sequence spaces and their $\beta$ duals. Let $E$ be a vector space of $X$ valued sequences which contains the space $c_{00}(X)$ of sequences which are eventually 0 . Let $Y$ be a Banach space and $L(X, Y)$ the space of continuous linear operators from $X$ into $Y$ with the strong operator topology $\tau$. The $\beta$ dual of $E$ with respect to $Y$ is defined to be

$$
E^{\beta Y}=\left\{\left\{T_{j}\right\} \subset L(X, Y): \sum_{j=1}^{\infty} T_{j} x_{j} \text { converges for every } x=\left\{x_{j}\right\} \in E\right\} .
$$

We write $T \cdot x=\sum_{j=1}^{\infty} T_{j} x_{j}$ when $T=\left\{T_{j}\right\} \in E^{\beta Y}$ and $x=\left\{x_{j}\right\} \in E$. Consider the triple $\left(E^{\beta Y}, E: Y\right)$ under the bilinear map $(T, x) \rightarrow T \cdot x$.

We consider the analogues of Propositions 7 and 8 for this triple.

Proposition 17. Suppose $K \subset E^{\beta Y}$ is $w\left(E^{\beta Y}, E\right)$ relatively sequentially compact. Then for every $j$,

(a) $\left\{T_{j}: T=\left\{T_{k}\right\} \in K\right\}$ is $\tau$ relatively sequentially compact.

Proof. The linear map $G_{j}: E^{\beta Y} \rightarrow L(X, Y), T \rightarrow T_{j}$, is $w\left(E^{\beta Y}, E\right)-\tau$ continuous so the result follows.

The space $E$ has the signed weak gliding hump property (signed-WGHP) if for every $x=\left\{x_{j}\right\} \in E$ and every increasing sequence of intervals $\left\{I_{j}\right\}$ there exists a subsequence $\left\{n_{j}\right\}$ and a sequence of signs $\left\{s_{j}\right\}$ such that the coordinate sum of the series $\sum_{j=1}^{\infty} s_{j} \chi_{I_{n_{j}}} x_{j} \in E$, where $\chi_{I}$ is the chacteristic function of $I$ and $\chi_{I} x$ is the coordinate product of $\chi_{I}$ and $x$. See [15] Appendix C for examples of spaces with signed-WGHP; for example, $l^{p}(X), c_{0}(X)$ have signed-WGHP. From Corollary 4 of [12] or Theorem 2.26 of [15], we have

Proposition 18. Assume $E$ has signed-WGHP and $K \subset E^{\beta Y}$ is $w\left(E^{\beta Y}, E\right)$ relatively sequentially compact. Then for every $x \in E$ the series

$$
\text { (b) } \sum_{j=1}^{\infty} T_{j} x_{j} \text { converge uniformly in } Y \text { for } T \in K \text {. }
$$

Conditions (a) and (b) are necessary conditions for relative sequential compactness. We now show that they are sufficient as in Theorem 9 .

Theorem 19. Let $K \subset E^{\beta Y}$ satisfy conditions (a) and (b). Then $K$ is $w\left(E^{\beta Y}, E\right)$ relatively sequentially compact.

Proof. Let $\left\{T^{k}\right\} \in K$. By (a) and the diagonalization method[7], there exists a subsquence $\left\{T^{n_{k}}\right\}$ such that $\tau-\lim _{k} T_{j}^{n_{k}}=T_{j} \in L(X, Y)$ for every $j$. Let $U$ be a closed neighborhood of 0 in $Y$ and $x \in E$. By (b). there exists $N$ such that $\sum_{j=n}^{p} T_{j}^{n_{k}} x_{j} \in U$ for $p>n \geq N, k \in \mathbb{N}$. Then $\sum_{j=n}^{p} T_{j} x_{j} \in U$ for $p>n \geq N$ so the series $\sum_{j=1}^{\in} T_{j} x_{j}$ converges or $T=\left\{T_{j}\right\} \in E^{\beta Y}$. Now

$$
\text { (\&) }\left(T^{n_{k}}-T\right) \cdot x=\sum_{j=1}^{M}\left(T_{j}^{n_{k}}-T_{j}\right) x_{j}+\sum_{j=M+1}^{\infty}\left(T_{j}^{n_{k}}-T_{j}\right) x_{j} .
$$

There exists $M$ such that the term on the right hand side of (\&) will belong to $U$ for every $k$ by (b). With this $M$ fixed the first term on the right hand side of (\&) will belong to $U$ for large $k$. Hence, $T^{n_{k}} \rightarrow T$ with respect to $\tau$ and $K$ is $w\left(E^{\beta Y}, E\right)$ relatively sequentially compact.

Note that the proof above also shows that $w\left(E^{\beta Y}, E\right)$ is sequentially complete when $E$ has signedWGHP and $X, Y$ are Banach spaces[15]. 


\section{References}

1. C. Bosch and C. Swartz, Functional Calculi, World Sci. Publ., Singapore, 2013.

2. A. Chen and R. Li, A Version of Orlicz-Pettis Theorem for Quasi-homogeneous Operator Space, J. Math. Anal. Appl., 373(2011), 127-133.

3. M. Cho, R. Li and C. Swartz, Subseries Convergence in Abstract Duality Pairs, Proy. J. Math., 33(2014), $447-470$.

4. M. Cho, R. Li and C.Swartz, The Banach-Steinhaus Theorem in Abstract Dua;ity Pairs, Proy. J. Math., 34(2015), 391-399.

5. N. Dunford and J. Schwartz, Linear Operators, Interscience, N.Y., 1958.

6. J. Diestel and J. Uhl, Vector Measures, Amer. Math. Surveys \#15, Providence, 1977.

7. J. Kelley, General Topology, Van Nostrand, Princeton, 1955.

8. R. Li and M. Cho, A General Kalton-like Theorem, J. Harbin Inst. Tech., 25(1992), 100-104.

9. R. Li and J. Wang, Invariants in Abstract Mapping Pairs, J. Austral. Math. Soc., 76(2004), 369-381.

10. R. Li and C. Swartz, An Abstract Orlicz-Pettis Theorem and Applications, Proy. J. Math., 27(2008), $155-169$

11. K. Musial, Topics in the Theory of Pettis Integration, Rend. D'Inst. Mat. Univ. Trieste, Trieste, 1991.

12. C. Stuart and C. Swartz, Uniform Convergence in the Dual of a Vector-Valued Sequence Space. Taiwan. J. Math., 7(2003), 665-676.

13. C. Swartz, Infinite Matrices and the Gliding Hump, World Sci. Publ., Singapore, 1996.

14. C. Swartz, Measure, Integration and Function Spaces, World Sci. Publ., Singapore, 1994.

15. C. Swartz, Multiplier Convergent Series, World Sci. Publ., Singapore, 2009.

16. F. Zheng, C. Cui and R. Li, Abstract Gliding Hump Properties in the Vector Valued Dual Pairs, Acta

Anal. Funct. Appl., 12(2010), 322-327. 